\title{
Cor Triatriatum Dexter and Patent Foramen Ovale Discovered Following Cardioembolic Stroke
}

\author{
Jacquelin Peck, MD, Nicholas Suraci, $M D^{1^{*}}$, Frederick Hasty, MD and Jayanard D'Mello, MD \\ Department of Anesthesiology, Mount Sinai Medica Center, USA \\ *Corresponding author: Nicholas Suraci, Department of Anesthesiology, Mount Sinai Medica Center, \\ 4300 Alton Road, Miami Beach, Florida 33140, USA, Tel: 305-674-2168
}

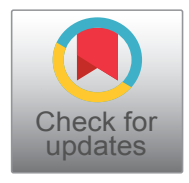

\begin{abstract}
Cor Triatriatum (CT) is a seldom-reported cardiac anomaly defined by the presence of an abnormal septation within the right or left atrium. Cor triatriatum usually remain asymptomatic until late adulthood, but depending on the severity of septation and location within the atrium the manifestations can vary in presentation. We present a case of cardioembolic cerebrovascular accident in a patient with cor triatriatum dexter and a patent foramen ovale. To our knowledge, no existing case report describes a cerebrovascular accident in a patient affected by right atrial cor triatriatum dexter.
\end{abstract}

\section{Abbreviations}

CT: Cor Triatrium; CTS: Cor Triatriatum Sinister; CTD: Cor Triatrium Dexter; CVA: Cerebrovascular Accident

\section{Introduction}

Cor triatriatum (CT) is a rare congenital cardiac anomaly with fewer than 250 cases reported within existing literature [1]. It is defined by the presence of an abnormal flap or septation within either the left atrium (cor triatriatum sinister (CTS)) or right atrium (cor triatriatum dexter (CTD). CT may exist as an isolated anomaly or in combination with additional congenital cardiac abnormalities. Most cases of CT are found in early infancy. However, few cases may remain asymptomatic until adulthood or be discovered post-mortem [2]. The septation created by CT impedes filling of the corresponding ventricle, with CTS and CTD symptomatically resembling mitral or tricuspid valvular stenosis respectively [3]. CT-related complications including pulmonary and venous hypertension, heart failure, atrial fibrillation, syncope, thrombus formation, systemic emboli, and sudden cardiac arrest have also been reported [4]. Several existing case reports describe cardioembolic stroke or cerebrovascular accident (CVA) in the context of left-atrial CTS [4-8]. However, to our knowledge no existing case report describes CVA in a patient affected by right atrial CTD. Here, we describe a case of cardioembolic CVA in a patient with CTD and a patent foramen ovale (PFO).

\section{Case}

A 36-year-old female with a past medical history of hyperlipidemia, peripheral scleroderma (morphea), renal cell carcinoma status post partial nephrectomy, childhood Ewing sarcoma status post chemotherapy, and recent cardioembolic stroke requiring intracranial thrombectomy by interventional radiology presented for cardiac surgery. The patient displayed no residual neurological deficits after cerebral thrombectomy. However, her stroke workup and evaluation were notable for a transesophageal echocardiogram (TEE) revealing severe non-rheumatic mitral regurgitation, mild to moderate non-rheumatic aortic incompetence, mild tricuspid regurgitation, patent foramen ovale, and right atrial cor triatriatum. The patient had a preserved left ventricular ejection fraction of $65 \%$ and no mass or thrombus were identified within the left or right atrium. Given aortic and mitral involvement, and the patient's history of scleroderma, she was evaluated by a rheumatologist who determined that the patient's scleroderma was isolated to the periphery without evidence of systemic

Citation: Peck J, Suraci N, Hasty F, D'Mello J (2020) Cor Triatriatum Dexter and Patent Foramen Ovale Discovered Following Cardioembolic Stroke. Int J Anesthetic Anesthesiol 7:103. doi.org/10.23937/23774630/1410103

Accepted: April 04, 2020: Published: April 06, 2020

Copyright: (C) 2020 Peck J, et al. This is an open-access article distributed under the terms of the Creative Commons Attribution License, which permits unrestricted use, distribution, and reproduction in any medium, provided the original author and source are credited. 


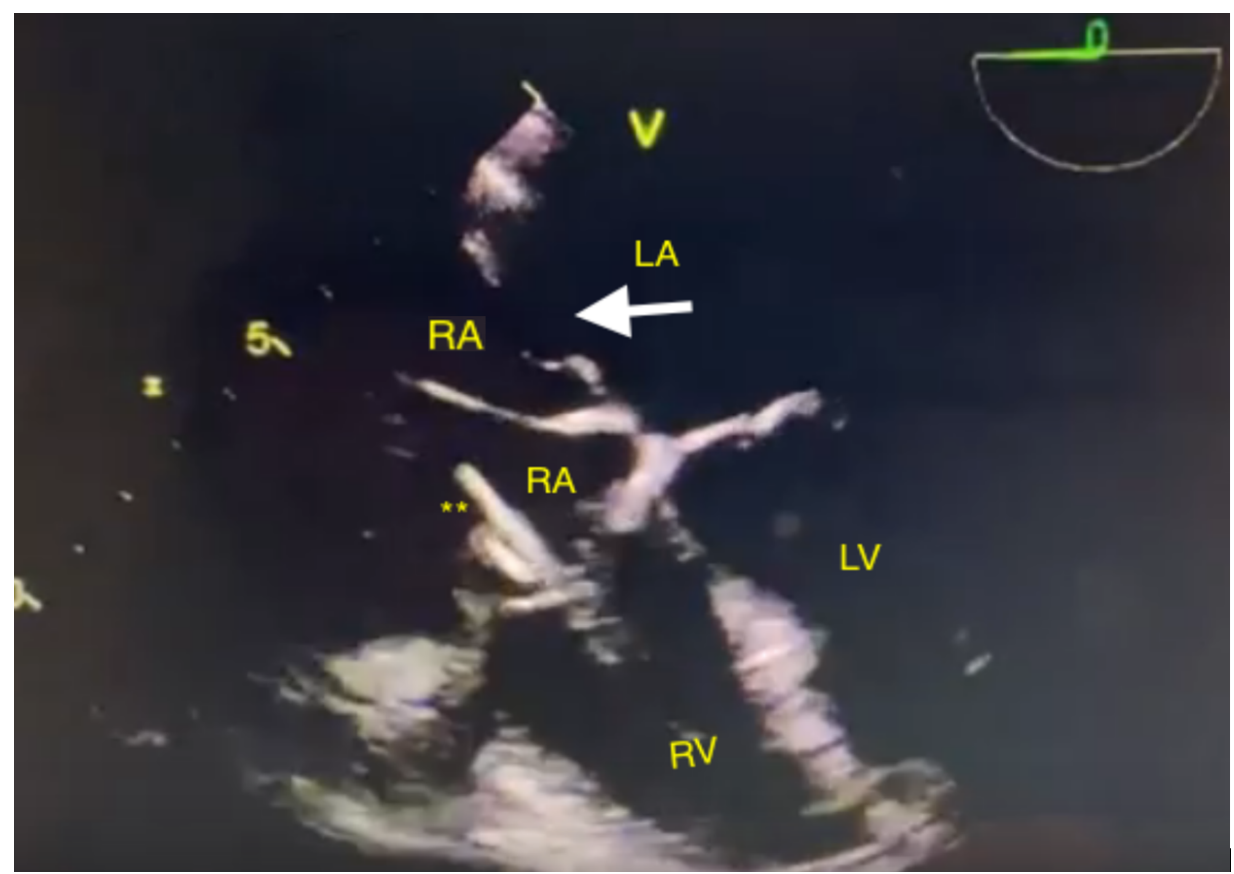

Figure 1: Transesophageal Echocardiogram demonstrating Cor Triatriatum Dexter with a patent foramen ovale. RA: Right Atrium; RV: Right Ventricle; LA: Left Atrium; LV: Left Ventricle

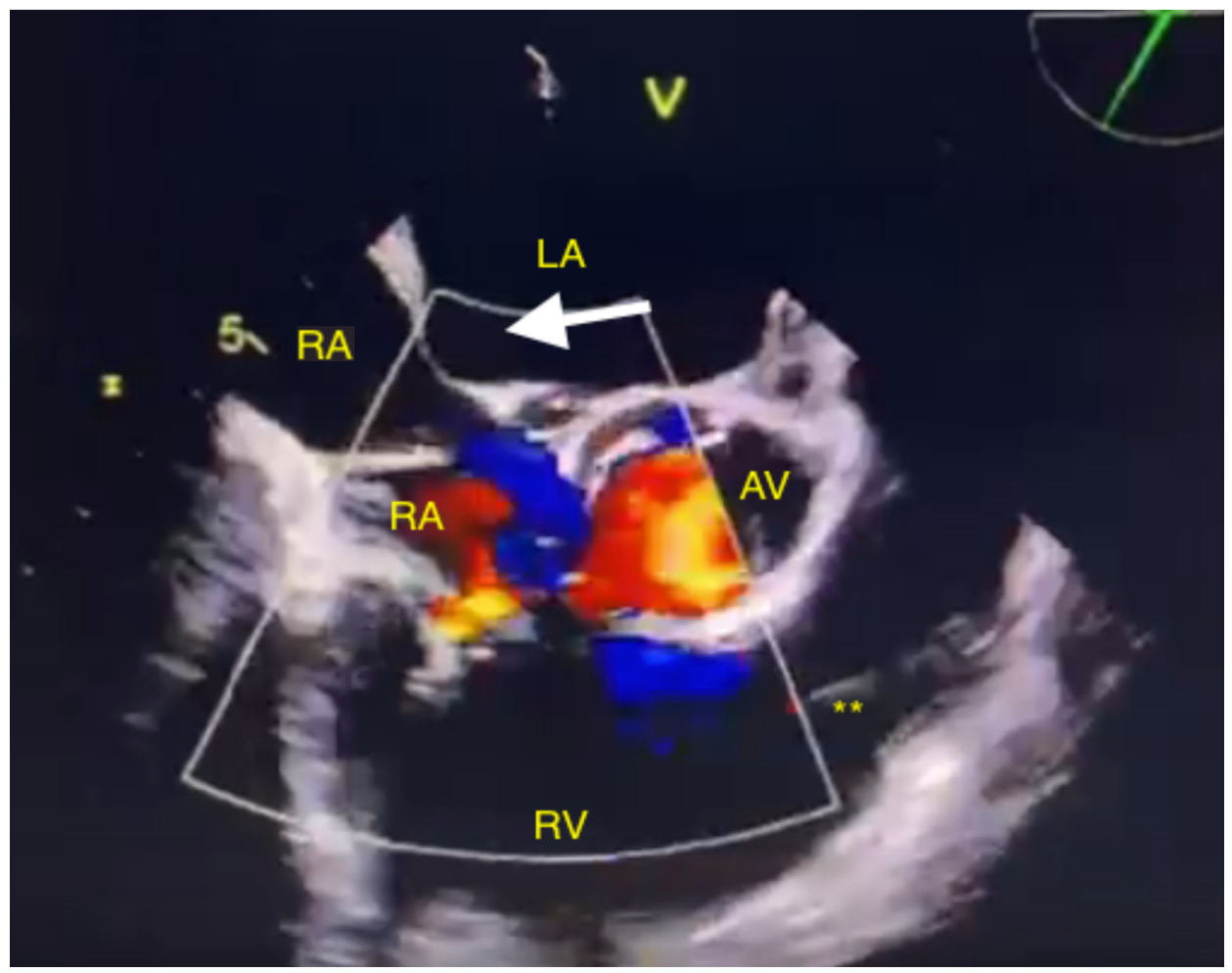

Figure 2: Transesophageal Echocardiogram with color flow doppler demonstrating Cor Triatriatum Dexter with blood flow into the right ventricle.

RA: Right Atrium; RV: Right Ventricle; LA: Left Atrium; LV: Left Ventricle

or cardiovascular involvement. The patient also denied a history of transient ischemic attack, syncope, chest palpitations or diagnosis of atrial fibrillation. Additional workup, including carotid arterial duplex and Holter monitor evaluation were unremarkable. Cardiogenic embolism was suspected, and the patient presented for minimally invasive mitral valve repair, resection of right atrial membrane, closure of the PFO, and ligation of the left atrial appendage, which proceeded uneventfully. TEE imaging (Figure 1, Figure 2 and Figure 3) were obtained by the patient's anesthesia providers following the induction of anesthesia and prior to surgical correction of her cardiac malformation.

The patient developed atrial fibrillation post-op- 


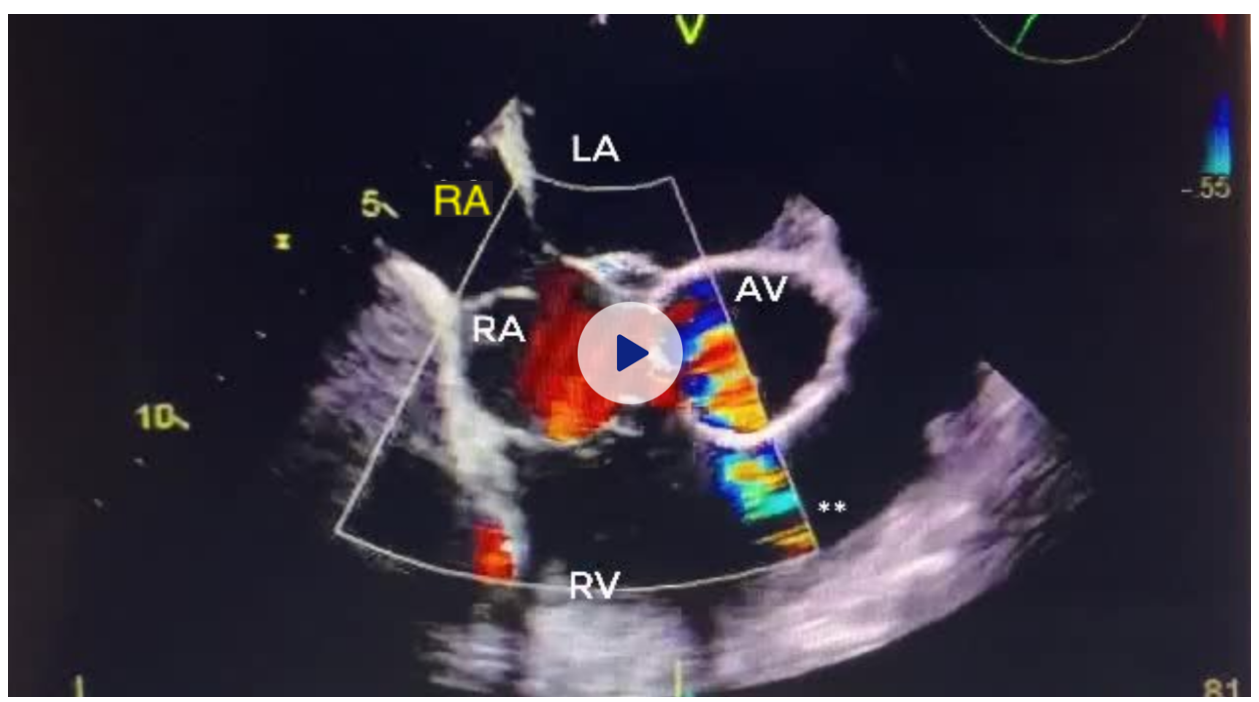

Figure 3: Transesophageal Echocardiogram with color flow doppler demonstrating Cor Triatriatum Dexter with blood flow between right atria. A small patent foramen ovale is also visible between right and left atria.

RA: Right Atrium; RV: Right Ventricle; LA: Left Atrium; LV: Left Ventricle

eratively and remains therapeutic on warfarin and Metoprolol. She recovered well post-operatively and was free from residual neurologic symptoms or subsequent cerebrovascular events at 2-month outpatient follow-up.

\section{Discussion}

Cor triatriatum is a rare congenital cardiac anomaly. Few affected patients progress to adulthood without symptomology or diagnosis. The embryologic basis of the disease remains unclear, however prevailing theories include atypical atrial tissue growth, incomplete fusion of septal tissues, and entrapment of atrial septum during cardiac development [3,9-11].

Classical CT is defined as isolated CT in the absence of other congenital cardiac malformations. However, in the Royal Children's Hospital's published 20 -year experience, $54 \%$ of patients ( $n=11$ out of 28 patients) presented with atypical $\mathrm{CT}$, which is $\mathrm{CT}$ with coexisting congenital cardiac malformations [1]. This finding was supported by the Mayo Clinic's published 50-year experience, which reports frequent diagnosis of CT with associated cardiac anomalies [3]. The most frequent coexisting cardiac anomalies included atrial septal defect $(n=7,28 \%)$, PFO $(n=6,24 \%)$, tricuspid regurgitation $(n=6,24 \%)$, and myxomatous mitral valve disease $(n=3,12 \%)$ [3]. Though CT remains rare, existing evidence suggests that our patient's simultaneous CT, PFO, and valvular disease may be consistent with known presentations of the anomaly. A review of existing literature reveals several reports of CT-related cardioembolic stroke [4-8]. These include cardioembolic stroke in an adult male with a TEE notable for CTS and a left atrial thrombus [4], a 17-year-old adolescent male who was diagnosed with CTS on TEE after presenting with an embolic cerebral infarct resulting in left hemiparesis [5], a case of CTA in a patient with CTS and left atrial thrombus identified on TEE [7], and a 55-year-old male with embolic CVA who was subsequently diagnosed with atrial fibrillation and had a TEE notable for left atrial enlargement, CTS, and a large thrombus visible within the left atrial accessory chamber [8]. Each case describes cardioembolic stroke in the context of CTS, aspecifically left-sided atrial malformation.

\section{Conclusion}

To our knowledge, no existing report describes a case of cardioembolic stroke with CTD, a right-atrial malformation. Our patient's PFO may have made passage of a right atrial thrombus to the left atrium, ventricle, and systemic circulation possible. However, the exact etiology of our patient's CVA remains unknown and her complex medical history should not be overlooked.

\section{Acknowledgements}

None.

\section{Conflicts of Interest}

None.

\section{Source of Support/Funding}

None.

\section{Declaration of Interest}

There an no financial and or personal relationships with other people or organizations that could inappropriately bias work.

\section{References}

1. Nelson Alphonso, Martin A Nørgaard, Andrew Newcomb, Yves d'Udekem, Christian P Brizard, et al. (2005) Cor triatriatum: Presentation, diagnosis and long-term surgical results. Ann Thorac Surg 80: 1666-1671. 
2. Jha A, Makhirja N (2017) Cortriatrium: A review. Semin Cartiothorac Vasc Anesth 21: 178-185.

3. Pankaj Saxena, Harold M Burkhart, Hartzell V Schaff, Richard Daly, Lyle D Joyce, et al. (2014) Surgical repair of cor triatrium sinister: The mayo clinic 50-year experience. Ann Thorac Surg 97: 1659-1663.

4. Spengos K, Gialafos E, Vassilopoulou S (2008) Ischemic stroke as an uncommon complication of Cor triatriatum. J Stroke Cerebrovasc Dis 17: 436-438.

5. Anil Minocha, Sanjeev Gera, Nishith Chandra, Atampreet Singh, Sanjay Saxena (2014) Cor triatriatum sinistrum presenting as cardioembolic stroke: An unusual cause of adolescent hemiparesis. Echocardiography 31: 120-123.

6. Kyung-Jun Park, II-Kwon Park, Jung-Ju Sir, Hyun-Tae Kim, Young-II Park, et al. (2009) Adult cor triatriatum presenting as cardioembolic stroke. Intern med 48: 1149-1152.
7. Sinorakis E, Arcanitakis S, Pantelis N, et al. (2013) Left atrium in cortiratiatum: Arrhythmogenesis and thrombogenesis leading to stroke. Int J Cardiol 168: 4503-4504.

8. Lee C, Son J, Shin D (2016) Cor triatriatum sinister - source of unusual thrombogenesis in mitral stenosis. Circ $\mathrm{J} 80$ : 256-257.

9. Gharagozloo F, Bulkley B, Hutchins, G (1977) A proposed pathogenesis of cor triatriatum: Impingement of the left superior vena cava on the developing left atrium. Am Heart $\mathrm{J}$ 94: 618-626.

10. Van Praagh R, Corsini I (1969) Cor triatriatum: pathologic anatomy and a consideration of morphogenesis based on 13 postmortem cases and a study of normal development of the pulmonary vein and atrial septum in 83 human embryos. Am Heart J 78: 379-405.

11. Fowler J (1881) Membranous band in the left auricle. Trans Pathol Soc Lond 33: 77-94. 